\title{
The Effect of Puromycin on Retention of Conditioned Cardiac Deceleration in the Goldfish ${ }^{1}$
}

\author{
W. MICHAEL SCHOEL and BERNARD W. AGRANOFF
}

\author{
Neuroscience Laboratory \\ University of Michigan \\ Ann Arbor, Michigan 48104
}

\begin{abstract}
The electrocardiogram was used to measure conditioned heart rate deceleration to a light-off signal paired with a punishing electrical shock in the goldfish. The learned response was retained for several days but was rapidly lost with extinction trials. Intracranial injections of puromycin administered just before or immediately after a training session did not appear to block formation of memory of this response. This result is discussed in relation to previous studies on the possible effects of puromycin on conditioned fear and instrumental learning.
\end{abstract}

\section{INTRODUCTION}

We have previously demonstrated that the intracranial injection of antibiotic blocking agents such as the protein synthesis inhibitor, puromycin, prior to or shortly after training produces severe deficits in the retention of a shock-avoidance task (Agranoff, Davis and Brink, 1966). Since fish injected prior to training can acquire the avoidance response, we have assumed that these agents affect retention by blocking the formation of "permanent," or long-term memory, but not short-term memory. Potts and Bitterman (1967) found that postsession injections of puromycin produced marked retention deficits in goldfish in a discriminative shuttlebox avoidance task as well as in a passive goal box avoidance task. In the shuttlebox, fish treated previously with puromycin had low responding levels and showed poor discrimination of positive and negative cue lights. In the passive avoidance apparatus, the amnestic effect was manifested by a relative loss of hesitancy in entering the goal box. The latter result was taken to indicate that the puromycin treatment did not interfere with performance of the avoidance response. On the basis of these findings, the authors concluded that puromycin impairs retention of

${ }^{1}$ Supported by grants from the NSF and NIMH. 
consolidation of conditioned fear. According to this hypothesis, when goldfish that have received an amnestic treatment are tested for retention of an avoidance response, they fail to avoid because the conditioned stimulus (CS) does not elicit fear. These studies do not, however, rule out the alternate possibility that puromycin interferes with consolidation of information more generally relevant to the instrumental behavior. Thus in the discriminitive shuttlebox avoidance, fish might have been fearful but unable to perform the appropriate response.

A more direct measure of emotionality should prove useful in further clarifying the mechanism of action of puromycin on memory. Several reports have shown that heart rate (HR) slowing in response to visual stimuli in the goldfish can be conditioned (Otis, Cerf and Thomas, 1957; McCleary, 1960; Hester, 1968). The present series of experiments utilizes this paradigm to investigate the putative effect of puromycin on conditioned fear. A single-session learning task was used so that the results from the present experiments could be related to previous experiments in this laboratory exploring the effects of amnestic agents on memory formation in the goldfish.

\section{METHOD}

\section{Subjects}

Common goldfish, $7.5-9.0 \mathrm{~cm}$ (18-25 g body weight) from snout to caudal peduncle, were obtained from Ozark Fisheries, Stoutland, Missouri. The fish were housed in 50-gal storage tanks for 1-3 weeks prior to an experiment. During this time they were fed every 3-4 days. After electrode implantation, they were placed in individual 1.5 liter plastic home tanks.

\section{Conditioning Apparatus}

A $22.5 \times 12.0 \times 15.5-\mathrm{cm}$ deep aluminum container filled with water to a depth of $5.5 \mathrm{~cm}$ was fitted with an aluminum cover $(23.0 \times 12.5 \times 16.0 \mathrm{~cm})$ to screen out both light and electrical noise. Water was changed after every third or fourth subject. Fish were restrained during a session by means of an inner clear Plexiglas box. This restraining box (see Fig. 1) did not immobilize the fish or interfere with full opercular excursion but did restrict gross movement and maintained the fish in a constant position and orientation relative to both the $\mathrm{CS}$ and the unconditioned stimulus (US).

The US, a $0.5-\mathrm{sec} 2.0-\mathrm{V}, 60-\mathrm{Hz}$ ac shock, was delivered through stainless-steel mesh electrodes $(11 \times 4.5 \mathrm{~cm})$ positioned on each side of the restraining box and separated from the fish by slotted, Plexiglas inserts, $0.6 \mathrm{~cm}$ thick. The CS light was a No. 330 lamp operated at $12 \mathrm{~V} \mathrm{dc}$ positioned behind the upper portion of a $19 \times 12-\mathrm{cm}$ piece of opal glass fitted to one side of the container. The upper $7.0 \mathrm{~cm}$ of this glass was made opaque so that the light was viewed indirectly by the fish. 


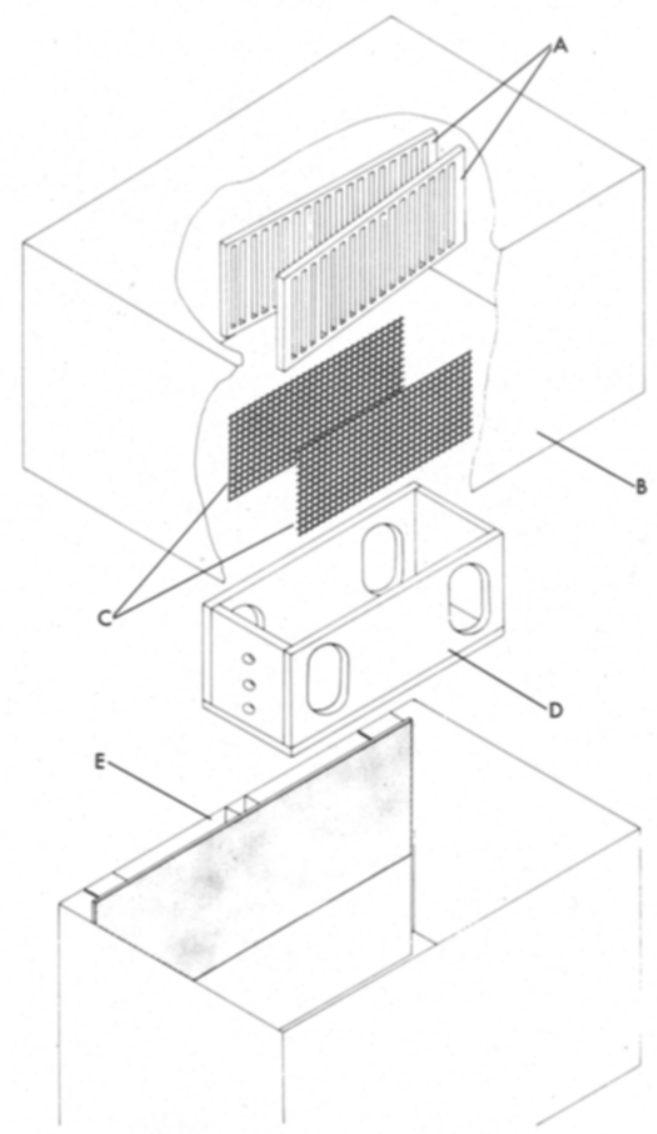

Fig. 1. Exploded view of the conditioning apparatus: $A$, Plexiglas inserts; $B$, cover; $C$, electrodes; $D$, restraining box; $E$, container, showing opal glass diffusing screen.

The onset and duration of CS and US were controlled by digital IC control equipment. This device also operated a polygraph stimulus marker pen and disconnected the electrocardiograph (ECG) recording electrodes from the amplifier during the US. Trials were initiated manually.

\section{ECG Recording}

Two fine, $0.076 \mathrm{~mm} \times 30-\mathrm{cm}$ enameled silver wire electrodes (Sigmund Cohn Corp., Mount Vernon, NY) were implanted in the fish. Approximately $0.5 \mathrm{~mm}$ of insulation was stripped from one end of each wire and this portion was then chlorided to reduce polarization artifacts by immersion for $5 \mathrm{sec}$ in $0.1 N \mathrm{HC} 1$ with the wire attached to the anode of a $1.5-\mathrm{V}$ dry cell. The circuit was completed with a carbon rod. To implant the electrodes, surgery was performed under tricaine methosulfate anesthesia (MS-222, Finquel, 
Ayerst). Two punctures for the electrodes were made in the fish's ventral midline with the tip of a 25-gauge hypodermic needle. The anterior electrode was placed in the pericardial cavity and the posterior electrode was positioned in the peritoneum (Fig. 2). Each electrode penetrated the fish to a depth of

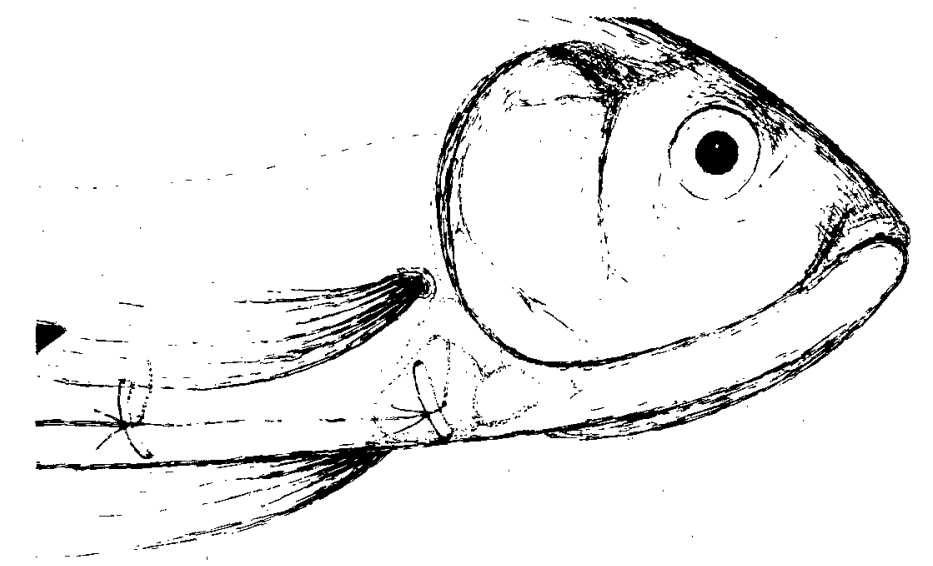

Fig. 2. Goldfish with ECG electrodes sutured in place. The anterior electrode is located within the pericardial cavity.

about $0.4 \mathrm{~cm}$. To secure the electrodes in position, lengths of the silver wire were knotted around the electrodes, fixed in position with a small amount of insl-X (INSL-X Products Corp., Yonkers, NY) and then sutured to the ventral integument of the fish. Amphenol subminiature pin connections (Amphenol No. 220-PO2) were soldered to the free ends for connection to the recording equipment. When a fish was not in the test apparatus, the pin connectors were embedded in a small Styrofoam float to impede fouling of the electrodes while it was swimming freely. To further prevent tangling of the leads and to reduce induction artifacts, the two recording leads were joined together with insl-X for all but $1 \mathrm{~cm}$ at each end.

The ECG signal was amplified by a PAR high impedance preamplifier (Princeton Applied Research model CR-4, Princeton, N.J.) and this amplified signal was then transmitted to both a monitor scope (Hewlett-Packard model $130 \mathrm{~A}$ ) and a Gilson polygraph (model M5P). This technique yielded generally artifact-free records (Fig. 3), although about $15 \%$ of animals were discarded during the 5-6 days of an experiment due to sickness or changes in electrode position leading to loss of signal.

\section{General Procedure}

On experimental Day 1 the electrodes were implanted, and each fish was placed in a 1.5-liter "home" tank painted black to reduce the ambient light level. The lid was left slightly ajar, so that some room light entered the container. On training days the fish was placed in the Plexiglas restraining box in the test 


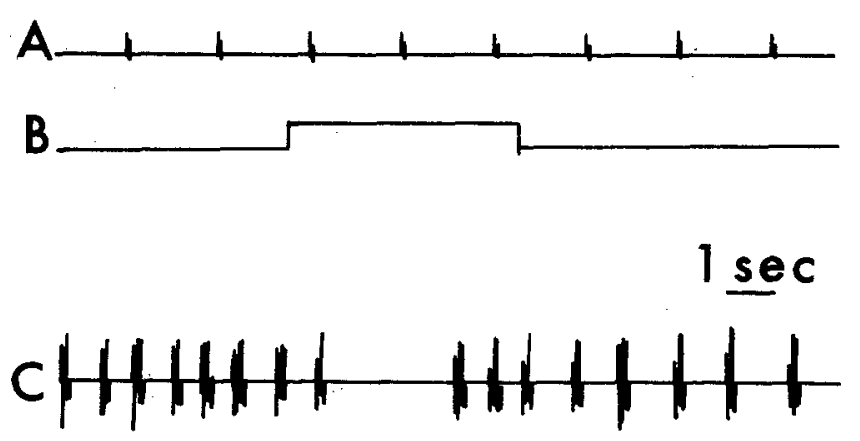

Fig. 3. Polygraph record of the effect of the CS on HR during the first extinction trial of a retention test session: $A$, time marker; $B$, CS marker; $C$ ECG.

apparatus and adapted for $15 \mathrm{~min}$ prior to the training session. The fish then received one of two types of training. Under one procedure, Desensitization, the fish received equal numbers of CS and US exposures in a quasirandom sequence with the following constraints: (1) no CS overlapped an US; (2) no stimulus occurred within $10 \mathrm{sec}$ of another stimulus; and (3) no stimulus followed another of the same type more than once. The same sequence was used for all fish. Under the second procedure, Conditioning, fish received classical conditioning trials with a CS duration of $5.5 \mathrm{sec}$, the last $0.5 \mathrm{sec}$ of which included the US. The CS used in this study was a 5 -sec "light-off" period. After training, fish were returned to their home tanks where they remained in semidarkness for the duration of the training-retention-test interval. During this period they were disturbed only for injections. To test for Retention, the fish were given the same number of CS presentations as in training, but without a US.

Heart rate was determined by measuring interbeat distances on the polygraph record (paper speed $=0.5 \mathrm{~cm} / \mathrm{sec}$ ) and then calculating the reciprocal of the mean value for two intervals. Interval A was a period of $5.0 \mathrm{sec}$ preceding the CS, and interval $\mathrm{B}$ was the $5.0 \mathrm{sec}$ after the CS onset. The two calculated rates were then used to derive the change in HR by the equation, percentage HR decrease $=[(A-B) / A] \times 100$. The puromycin treatment in all cases consisted of an intracranial injection of $195 \mu \mathrm{g}$ " of puromycin dihydrochloride (Nutritional Biochemicals, Cleveland) in $15 \mu \mathrm{l}$ of saline, administered via a Hamilton syringe fitted with a 30-gauge needle. This amount is somewhat greater than was previously used in smaller goldfish and produced $62 \%$ inhibition of brain protein synthesis.

\section{EXPERIMENT I}

This study was performed to confirm that parameters developed in preliminary studies would produce a $\mathrm{CR}$ in a single session which would be 
retained for at least $48 \mathrm{hr}$. Electrodes were implanted on experimental Day 1, and on Day 2 the fish received 20 uncoupled presentations of the CS and US. On Day 3 the animals were divided into two matched groups, on the basis of their response to the CS in the preceding session. One group was given an additional desensitization session on this day to determine possible nonspecific effects of the US on the response, and the second was given 20 conditioning trials. The fish remained undisturbed in their home tanks until the retention test session on Day 5. Conditioning and test sessions were $30 \mathrm{~min}$ long with a variable intertrial interval $(45-135 \mathrm{sec})$. Desensitization sessions were also $30 \mathrm{~min}$ and the interstimulus interval varied between 25 and $65 \mathrm{sec}$.

\section{Results}

The fish usually struggled briefly when placed in the apparatus but were quiet long before the end of the 15-min adaptation period. The basal HR was highly variable, both between fish and from day to day in the same fish, ranging from 15 to 80 beats $/ \mathrm{min}$. The within-session basal $\mathrm{HR}$ was much less variable, usually less than $50 \%$.

The two groups of fish ( $n=15$ each), while matched with respect to their Day-2 performance, showed distinctly different response patterns (Fig. 4)

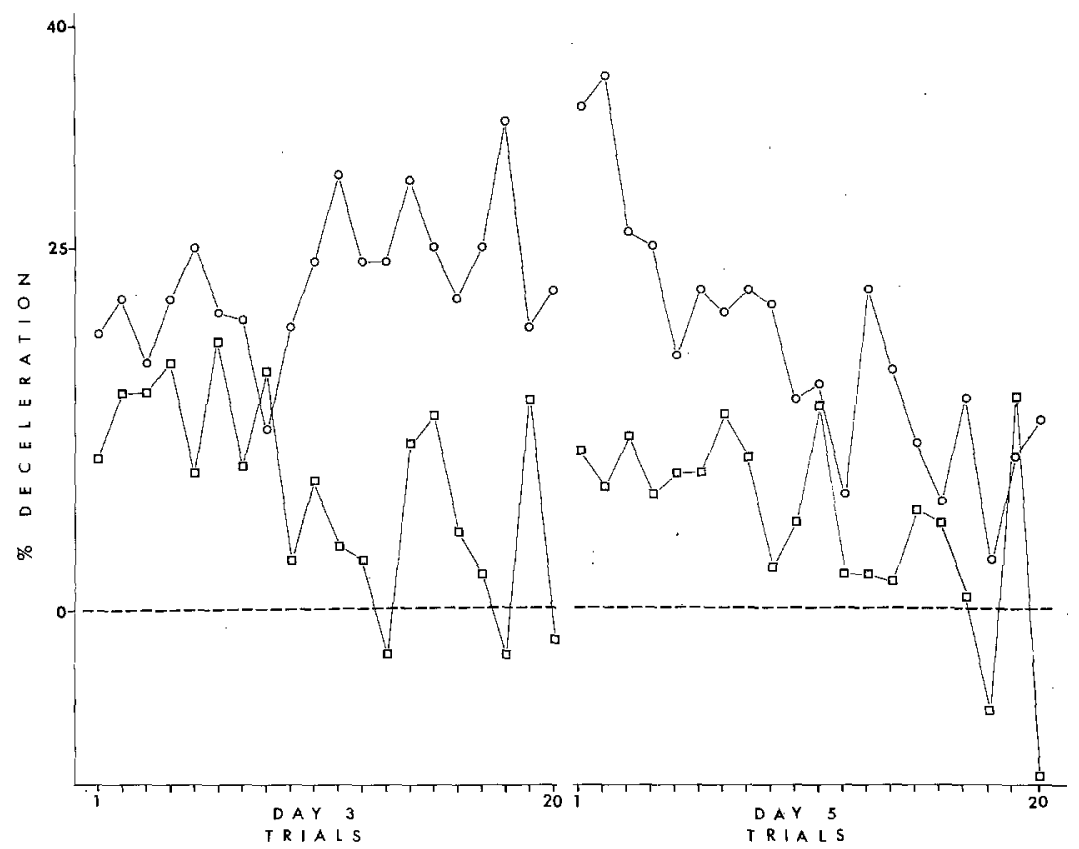

Fig. 4. Mean effect of the CS on HR of two groups of goldfish, one receiving conditioning $(O)$ and the other receiving desensitization training ( $\square$ ). Fish were trained on Day 3 and tested for retention on Day 5. 
on Day 3 and Day 5 [Groups, $F(1,28)=13.272, \quad P<0.005$; Groups $X$ Day $X$ Trials, $F(19,532)=1.826, P<0.025]$. These results indicated that conditioning, as distinct from sensitization, had occurred. During the training session (Day 3), the degree of HR slowing to the CS increased in the conditioned fish and decreased in the ones that continued to receive desensitization trials [Groups $X$ Trials, $F(19,532)=2.172, P<0.01$ ] . Further evidence of learning is shown by comparisons between training and retention test performance within each group. While there was a large difference in performance of the conditioned fish in these two sessions [Day $\times$ Trials, $F(19,266)=2.969, P<0.001]$, the response pattern of the control group in the retention test did not differ from that in the training session (Day $X$ Trials, $F=0.536$ ). In the group given conditioning trials, the responses in the last session were quite large initially, then declined with repeated exposures to the CS, indicating the extinction of the learned behavior [Trials, $F(19,266)=3.139, P<0.001]$. The control fish responses to the CS, which were not as large [Groups, $F(1,28)=15.767, P<0.001]$ also declined within the session [Trials, $F(19,266)=1.647, P<0.05$ ] as they had on Day 3 .

\section{EXPERIMENT II}

This experiment was performed to investigate the possibility that the conditioned cardiac response was susceptible to the amnestic effects of puromycin. Two groups of fish ( $n=18$ each) were conditioned and tested using the procedure described in Expt I. One of the groups was injected intracranially with $195 \mu \mathrm{g}$ of puromycin immediately after training, and the other was injected with the drug $4 \mathrm{hr}$ after training. This interval was chosen to control for possible toxic drug effects (Casola et al, 1968).

\section{Results}

The performance of both groups (Fig. 5) closely resembled that of the conditioning group in the preceding experiment [Day $\times$ Trials, $F(19,646)=4.886, P<0.001]$. Conditioned responses increased over trials on Day 3 [Trials, $F(19,646)=2.006, P<0.025]$. On Day 5 , the responses to the CS were initially large but decreased with repeated presentations [Trials, $F(19,646)=4.267, P<0.001]$ as they had in the first experiment. The absence of a group difference in the retention test [Groups, $F=0.006$; Groups $X$ Trials, $F=0.713$ ] indicated that puromycin did not produce a significant amnestic effect.

\section{EXPERIMENT III}

Since retention test performance in the previous experiment suggested that puromycin had little effect on the CR either when injected immediately 


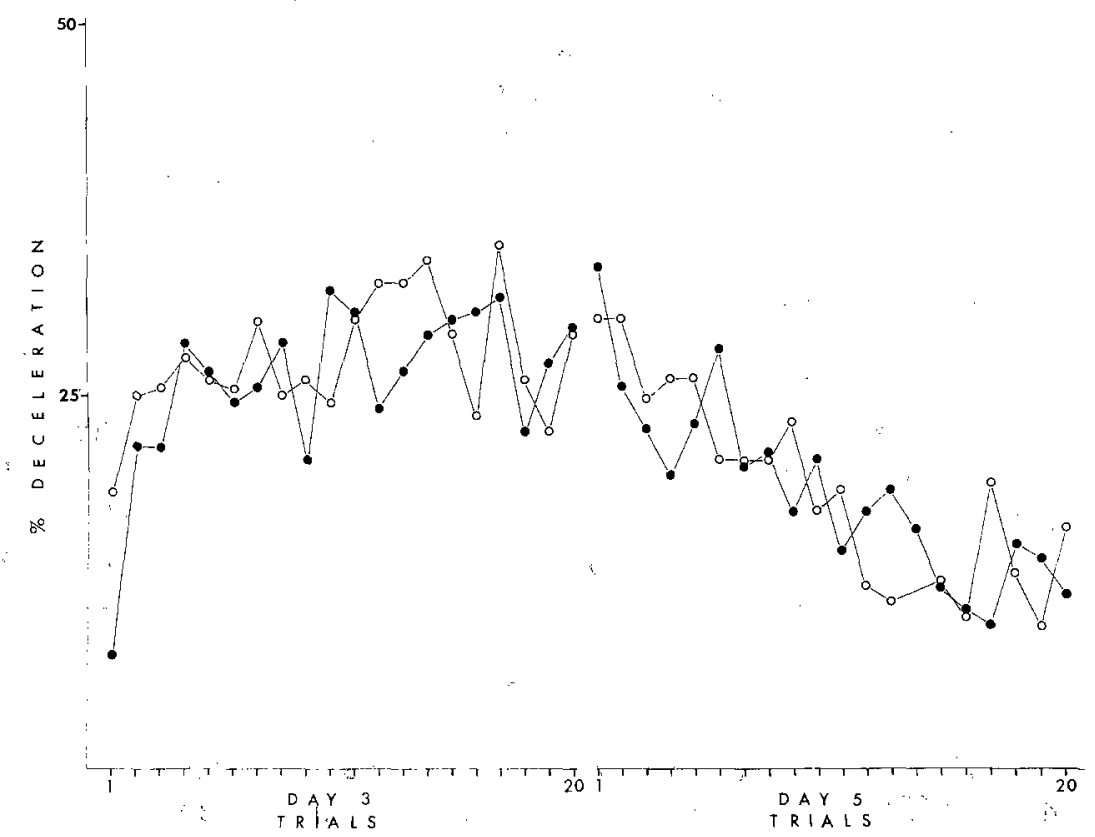

Fig. 5. Acquisition and extinction of HR response to a CS in two groups of goldfish injected with puromycin either immediately after $(\bullet)$ or $4 \mathrm{hr}$ after conditioning (O).

after the end of a training session or $4 \mathrm{hr}$ after training, a further experiment was designed to determine whether the lack of a demonstrable amnestic effect could be attributed to a rapid consolidation of memory, i.e., that permanent memory had formed prior to the injection of puromycin after the twentieth trial. Twenty-five fish were trained as in Expts I and II, but they received $195 \mu \mathrm{g}$ of puromycin intracranially $30 \mathrm{~min}$ before training on Day 3 .

Since the lack of a difference between the two groups in Expt II might have been attributable to a prolonged susceptibility of the learned response to puromycin extending beyond $4 \mathrm{hr}$ after the training session, a second group of 25 fish was injected with puromycin $24 \mathrm{hr}$ after training. Retraining was performed on Day 6 to assure sufficient time for the decay of short-term memory (Davis and Agranoff, 1966).

To balance possible effects of the injection procedure, the group injected with puromycin before training also received an intracranical injection of saline $24 \mathrm{hr}$ after training. Conversely, the group that was to receive puromycin $24 \mathrm{hr}$ after training was in addition injected with saline prior to training. Each group thus received one puromycin injection and one saline injection. 
Results

Generally the results of this experiment (Fig. 6) conformed with those of Expt II $[$ Day $\times$ Trials, $F(19,912)=10.030, P<0.001$ : Trials (Day 3),

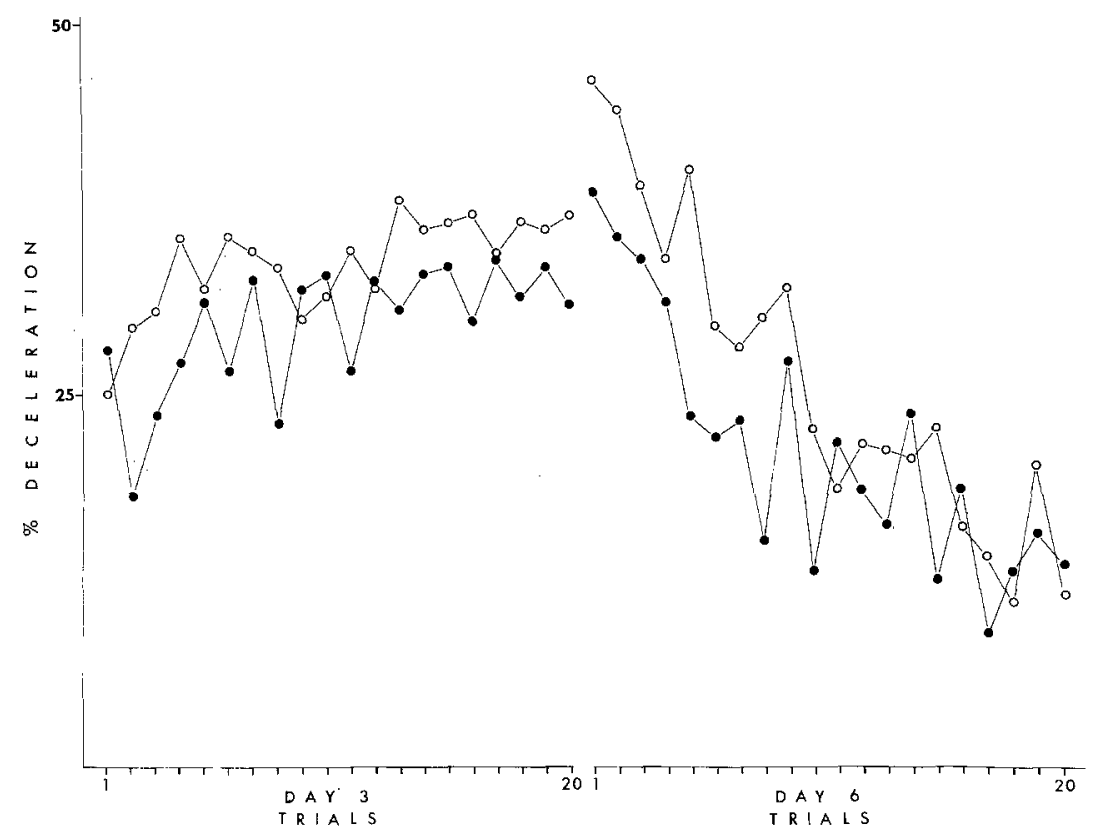

Fig. 6, Acquisition and extinction of HR response to a CS in two groups of goldfish injected with puromycin either $30 \mathrm{~min}$ prior to (•) or $24 \mathrm{hr}$ after the conditioning session $(O)$.

$F(19,912)=1.533, P<0.05$; Trials (Day 6), $F(19,912)=10.460, P<0.001]$. On Day 3, there were no observable effects of the injections on acquisition. The apparent slower HR of the experimental fish on most training trials did not occur consistently in all animals and did not approach statistical significance [Groups, $F(1,48)=1.338, P>0.10]$. Once more, the drug treatment did not affect retention of the conditioned response [Groups, $F(1$, $48)=2.038, P>0.10$; Groups $X$ Trials, $F=0.942]$. These results support the conclusion that puromycin does not block formation of memory of the conditioned HR response.

\section{EXPERIMENT IV}

This experiment explored the possibility that the absence of an amnestic effect of puromycin in the previous experiments was due to overtraining. As previous reports have shown the amount of training to be an important determinant of amnestic effects (Barondes, 1970), the number of trials was 
reduced to 10 in a 20 -min session (intertrial interval, 60-180 sec). To rule out the possibility that the desensitization training on Day 2 had some nonspecific activating effect which somehow obscured the amnestic effect of the drug, this procedure was omitted from the experiment. As these conditions were notably different from those used in the preceding experiments, desensitization control groups were included (interstimulus interval, 30-120 sec). An additional purpose of this experiment was to investigate the possibility that puromycin might influence possible changes in performance produced by the -desensitization procedure. Four groups of 25 fish each received a conditioning or desensitization session on Day 2 in combination with an intracranial puromycin injection either immediately or $24 \mathrm{hr}$ after the training session. The retention test session on Day 5 consisted of $10 \mathrm{CS}$ presentations (20-min session; intertrial interval, 60-180 sec).

\section{Results}

The conditioning groups and the desensitization groups began training with similar responses to the CS, but within the 10-trial session diverged rapidly (Fig. 7) [Conditioning-Desensitization $X$ Trials, $F(9,864)=11.656$,
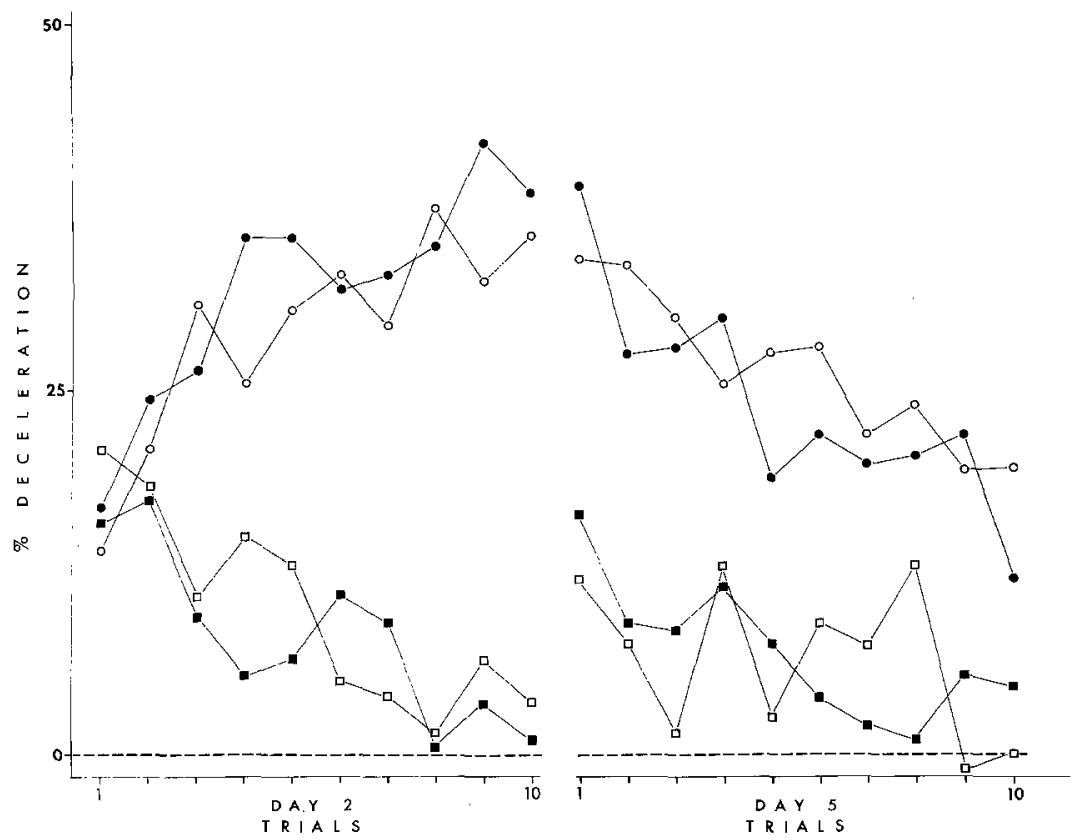

Fig. 7. Mean HR response in the training (Day 2) and retention test (Day 5) session of four groups of goldfish given either conditioning or desensitization training and injected with puromycin either immediately or $24 \mathrm{hr}$ after the training session. Conditioning zero delay puromycin; $O$ conditioning, 24-hr delay puromycin; desensitization, zero delay puromycin; $\square$ desensitization 24 -hr delay puromycin. 
$P<0.001$; Trials (Conditioning), $F(9,432)=5.717, P<0.001$; Trials (Desensitization), $F(9,432)=6.631, P<0.001]$. On testing on Day 6 , the conditioned group demonstrated the learned response initially and extinguished rapidly [Day $\times$ Trials, $\quad F(9,432)=11.180, \quad P<0.001$; Trials, $\quad F(9,432)=5.482$, $P<0.001]$. By contrast, the responses of the desensitization groups on Day 6 which also decreased within the session [Trials, $F(9,432)=1.974, P<0.05$ ] were smaller than those of the conditioned fish [Conditioning-Desensitization, $F(1,96)=61.240, P<0.001]$ but were not significantly different from the responses on Day 2 [Day $X$ Trials, $F(9,432)=1.430, P>0.10$ ]

There was again no difference in performance between the drug treatment groups on the acquisition day, indicating that they had been well matched [Drug Treatment $X$ Trials (Conditioning), $F=0.703$; Drug Treatment $X$ Trials (Desensitization), $F(9,432)=1.097, P>0.10]$. The absence of a drug effect on retention [Drug Treatment $X$ Trials (Conditioning), $F=0.935$; Drug Treatment $X$ Trials (Desensitization), $\quad F(9,432)=1.243, \quad P>0.10]$ suggests that puromycin injections immediately after training did not affect memory formation in this experiment.

\section{DISCUSSION}

Since puromycin did not block retention of conditioned cardiac deceleration under the conditions of our experiment, the results may be at variance with the interpretation of Potts and Bitterman (1967) that puromycin produces amnesia in the goldfish via a block of conditioned fear. The effect of puromycin in their experiments may, therefore, be more readily interpreted to indicate that puromycin disrupts some more complex aspect of behavior and that the lower rate of response to both the negative and positive stimuli in their experiments may indicate a partial failure of the fish to acquire the learned response. Huber and Longo (1970) reported that puromycin produces a deficit in the retention of conditioned body movement, a response interpreted to be an index of fear. In that study, a temporal susceptibility gradient to the drug was not found, so that motor impairment, sickness, and other nonspecific effects of puromycin cannot be ruled out. Their results appear to be similar to those of Mendoza and Adams (1968) and Hine and Paolino (1970) in which another amnestic agent (electroconvulsive shock) did not block retention of HR deceleration in the rat. In contrast, Davis and Holmes (1971) have reported a time-dependent effect of electroconvulsive shock on another presumed measure of conditioned fear, the conditioned inhibition of respiration in goldfish.

The varied effects of amnestic agents, both in goldfish and in higher animals, may be accounted for by some explanation other than distinction between classically conditioned and instrumental behavior. It is possible, for example, that avoidance responding is more susceptible to an amnestic agent than is the conditioning of an autonomic response simply because it is 
neurally more complex. If an agent such as puromycin reduced the efficacy of every synapse by $1 \%$, then in a series polysynaptic pathway of 10 synapses, the net reduction of signal would be $10 \%\left(0.9^{10}\right)$. A $1 \%$ reduction in a network of 100 synapses would result in a $65 \%$ signal reduction $\left(0.9^{100}\right)$. From a more general standpoint, we can visualize a complex neural signal that must be distinguished or a highly coordinated motor response which must be organized, and in which a given amount of behavioral, electrical, or chemical "noise" would generate more interference than in a less complex process. In this way, amnestic agents would be expected to have much greater effects on behaviors that require more neural events. This interference may be the blocking of chemical processes necessary for the formation of memory or an alteration of the chemical and/or electrical events leading to its formation. Regardless of the mechanism, it is significant that a learning and memory paradigm has been found in the goldfish which appears not to be susceptible to an inhibitor of protein synthesis. The lack of effect on sensitization and acquisition is expected and consonant with results found in shock-avoidance training. The lack of effect on conditioned heart-rate deceleration may indicate that a specific action of puromycin on conditioned fear is not likely to mediate its effect in more complex behaviors. A question remains concerning the relevance of cardiac deceleration to fear. Both deceleration and acceleration have been reported in homeotherms under conditions of classical and instrumental conditioning (Zeaman and Smith, 1965; Anderson and Brady, 1971): The possibility that deceleration indicates increased attentiveness (Billings and Shepard, 1910) may well pertain to homeotherms and poikilotherms alike. In the fish, deceleration is by far the prevalent heart-rate response. This may be related to the fact that the vagus mediates most if not all direct central cardiac control in the fish (Johansen, 1971). More direct evidence for a possible relationship of cardiac deceleration to conditioned fear and to concomitants of instrumental learning may derive from experiments which dissociate the effects of puromycin on the emotional response and instrumental learning in the same animal.

\section{REFERENCES}

Anderson, D. E., and Brady, J. V. (1971). Preavoidance blood pressure elevations accompanied by heart rate decreases in the dog. Science 172, 595-597.

Agranoff, B. W., Davis, R. E., and Brink, J. J. (1966). Chemical studies on memory fixation in goldfish. Brain Res. 1, 303-309.

Barondes, S. H. (1970). Cerebral protein synthesis inhibitors block long-term memory. Int. Rev. Neurobiol. 12, 177-205.

Billings, M. L., and Shepard, J. F. (1910). The change of heart rate with attention. Psychol. Rev. 17, 217-227. 
Casola, L., Lim, R., Davis, R. E., and Agranoff, B. W. (1968). Behavioral and biochemical effects of intracranial injection of cytosine arabinoside in goldfish. Proc. Nat. Acad. Sci. U.S. 60, 1389-1395.

Davis, R. E., and Holmes, P. A. (1971). ECS-produced retrograde amnesia of conditioned inhibition of respiration in cataleptic goldfish. Physiol. Behav. 7, 11-14.

Hester, F. J. (1968). Visual contrast thresholds of the goldfish (Carassius auratus). Vision Res. 8, 1315-1335.

Hine, B., and Paolino, R. M. (1970). Retrograde amnesia: production of skeletal but not cardiac response gradient by electroconvulsive shock. Science 169, 1224-1226.

Huber, H., and Longo, N. (1970). The effect of puromycin on classical conditioning in the goldfish. Psychon. Sci. 18, 279-280.

Johansen, K. (1971). Comparative physiology: Gas exchange and circulation in fisshes. Ann. Rev. Physiol. 39, 569-612.

McCleary, R. A. (1960). Type of response as a factor in interocular transfer in the fish. $J$. Comp. Physiol. Psychol. 53, 311-321.

Mendoza, J. E., and Adams, H. E. (1969). Does electroconvulsive shock produce retrograde amnesia? Physiol. Behav. 4, 307-309.

Otis, L. S., Cerf, J. A., and Thomas, G. J. (1957). Conditioned inhibition of respiration and heart rate in the goldfish. Science 126, 263-264.

Potts, A., and Bitterman, M. E. (1967). Puromycin and retention in the goldfish. Science 158, 1594-1596.

Zeaman, D., and Smith, R. W. (1965). Review of some recent findings in human cardiac conditioning. In W. F. Prokasy (Ed.), "Classical Conditioning," New York: Appelton-Century-Crofts. 